Amharic words in the text appear in their singular form.

abbat: father

adegegna bozene: dangerous vagrants

ammet bal: holiday

Arada: name of the inner city

Arada: a smart person

arakie: strong local brandy

bale-wuqabe: person with a relationship with a spirit (wuqabe)

bammilo: thug realism

bergo: cheap hotel

birr: Ethiopia’s currency

čaqquli: hurry

čebeta: squeezed

čebu: stealing after hitting the victim on the back of the neck

chewa: good, genuine guy

č'iggir: problem

činqet: stress, worry

čista: broke

COOPI: Cooperazione Internazionale I (nongovernmental organization)

debtera: religious expert

Derg: "the committee"; the military junta that ruled Ethiopia between 1974 and 1991

duriye: thug

EC: Ethiopian calendar

EPRDF: Ethiopian People's Revolutionary Democratic Front

EPRP: Ethiopian People's Revolutionary Party

faranjii: foreigner

farra: country bumpkin

ganja: cannabis

gashe: term of address to an elderly man 
geta: lord

GTZ: German Technical Cooperation (now GIZ)

gujbet: broke

habesha: Ethiopian

hardegna: hard man / gangster

hilinna: conscience

idil: chance

joffe: a hustler

IMF: International Monetary Fund

injera: Ethiopian pancake

inqisiqase: move around

khat: plant with a mild narcotic effect chewed across East Africa and the Middle East

khat bet: khat chewing house

kibur: respect

krar: Ethiopian lyre

listro: shoeshine boy

manfas: spirit

Meison: Amharic acronym of All Ethiopia Socialist Movement (AESM)

mella: a way of getting by

mirča: choice

NEBE: National Electoral Board of Ethiopia

NGO: nongovernmental organization

ruffiano: sycophant

şäşät: repentance

sefer: neighborhood

taj bet: honeywine drinking house

tanqway: sorcerer / fortune teller / medium

tara askabari: queue keepers, minibus touts

tigab: arrogance

TPLF: Tigray People's Liberation Front

video bet: video house / video parlor

yeArada kwankwa: Arada's slang

yemot midib: "those who are destined to die"; a gang in Arada 


\section{THE ACT OF LIVING}


